\title{
New Efficient PAPR Reduction Schemes in LTE OFDM Systems
}

\author{
Saleem Nokaiee, Mohammed M. Alkhawlani \\ University of Science and Technology \\ Yemen \\ snokaiee@yahoo.com \\ m.alshadadi@ust.edu
}

\begin{abstract}
Orthogonal frequency division multiplexing (OFDM) is a prominent, new multicarrier modulation technology used with most $4 G$ wireless communication systems. It was configured with MIMO to transmit very high rates of digital information with enhanced performance and speed. However, OFDM faced high peak to average power ratio (PAPR) issue at the transmitter. In this paper, a new companding method called advanced exponential companding (AEC) is proposed to provide better PAPA and BER performance than standard exponential companding (EC) and Mu-law companding while keeping constant average power before and after companding process. In addition, a new efficient hybrid scheme that is containing Zaddoff Chu Matrix Transform (ZCT) precoding and the proposed AEC companding is developed. The new scheme lessens the signal distortion caused by AEC companding and gives a great improvement in both PAPR \& BER performance in comparison with AEC in both LTE SISO-OFDM and LTE MIMO-OFDM systems. It produces high PAPR performance with slight SNR loss at 10^-3 BER against ZCT precoding with LTE MIMO-OFDM system. It offers the best results in terms of PAPR \& BER against those related existing hybrid methods, namely; discrete hartley transform (DHT) precoding + Mu-law companding, Zadoff -chu matrix transform (ZCT) + Mu-law companding, Walsh Hadamard Transform (WHT) precoding + Mu-law companding, Walsh Hadamard Transform (WHT) precoding + exponential companding (EC), and discrete cosine transform (DCT) precoding + Mu-law companding for LTE SISO-OFDM system.
\end{abstract}

Keywords: Advanced Exponential Companding (AEC), Zaddoff Chu Matrix Transform (ZCT) Precoding, Orthogonal Frequency Division Multiplexing (OFDM), Peak to Average Power Ratio (PAPR)

Received: 2 February 2019, Revised 20 May 2019, Accepted 28 May 2019

DOI: $10.6025 / \mathrm{stj} / 2019 / 8 / 2 / 39-53$

(C) 2019 DLINE. All Rights Reserved

\section{Introduction}

The LTE is identified by 3GPP to satisfy next decades supplementary increasing people needs for high data rates broadband services like video conferencing, online gaming and high audio/video streaming. LTE has used Orthogonal Frequency Division

Signals and Telecommunication Journal Volume 8 Number 2 September 2019 
Multiplexing (OFDM) technology for transmission which is one of the prominent recent techniques utilized with all modern wireless \& wired communication systems. OFDM is a new modulation method presenting low Inter Symbol Interference (ISI), obstruction in accordance with multipath fading channels, excellent spectral performance and easy receiver structure. However, one of the key drawbacks of OFDM based systems is the excessive PAPR issue that happens because of vast envelope oscillations in the transmit OFDM. The high PAPR signal either need a highly linear high-power amplifier (HPA) where power amplifier (PA) with substantial straight range is costly, bulky $50 \%$ of the size of a transmitter lies, hard to produce and has poor efficiency or cut by HPA which introduce in band distortion and out of band radiation into the system prompting an expansion in BER performance. Accordingly, a better arrangement is than discover lessening techniques with some processing of the OFDM signal itself. Many methodologies have been recommended to diminish PAPR in OFDM based frameworks. The present techniques are characterized into two sorts: signal scrambling methods and signal distortion schemes. Signal scrambling techniques do not twist the structure of the OFDM signal and no spectral regrowth occur. These schemes incorporate coding schemes [1-3] which cause no distortion and make no out of band radiation, but experience from bandwidth efficiency as the code rate is lessened and also suffer from complexity to discover best codes for an expansive number of subcarriers, both PTS [4-9] and SLM [10-15] schemes accomplish significant PAPR reduction at the expense of high computational complexity and data rate loss (side information). Signal distortion methods introduce spectral regrowth or distort the spectrum of the signal and transmit a PAPR signal with no extra data. These techniques consisting clipping and filtering scheme [16-20] and compandings methods [1], [2], [21-23], [24] which are the famous signal distortion schemes. The clipping-filtering scheme prompts a significant increment in BER performance at the receiver side. The compandings considered as special clipping techniques offering good PAPR lessening with better BER performance, low implementation complexity and no bandwidth expansion. Jiang et al. [1] proposed the outstanding exponential companding (EC) scheme. By converting the Rayleigh distributed OFDM signal into a signal with uniform distribution, EC technique can decrease PAPR enormously. In addition, EC method can present design freedom in the PAPR reduction with a flexible parameter which decide the distribution of the companded signals. Zainb AlHashmi [24] proposed six new compandings; Rooting Companding (RCT), New Error Function Companding (NEFC), Cosine Companding (COS), Absolute Exponential Companding (AEXP), Tangent Rooting Companding (TanhR), and Logarithmic Companding (LogR) providing better performance than Mu-law Companding, and the best performance in terms of PAPR \& BER has been achieved by AEXP companding. Jamadar and Patil [21] used nonlinear companding (NLC) and Exponential Companding with MIMO-OFDM and the improvement in PAPR and BER with nonlinear companding (NLC) was better than exponential companding (EC). Ramaswamy and Reddy [22] suggested iterative piecewise companding transform (IPCT) approach for PAPR reduction in LTE MIMO-OFDM and compare the approach with Iterative Linear Transform Approach (ILST) and Iterative Two Piecewise Companding Transform (ITPWC). The evaluation of performance was done and demonstrated that IPCT earned very low BER and PAPR among the above-mentioned methods with consuming low computations which make it widely used for 4G, WLAN and WiMAX applications. Das et al. [23] proved that EC presents higher performance than clipping based Active Constellation Extension (CCB-ACE) method. Regarding BER performance improvement, some researchers have assumed hybrid methods which integrate precoding and companding [25], [26], [27]. Bindu and Chandrika [25] assumed Discrete Cosine Transform (DCT) precoding combined with companding achieving enhanced PAPR reduction. Rao et al. [26] proposed Piecewise Linear Companding (PLC) combined with three precoder schemes; Discrete Hartly Transform (DHT), Wash Hadmard Transform (WHT) and Discrete Cosine Transform (DCT) and the PLC with DHT method achieved the best BER\&PAPR performance among others.

In this paper, two PAPR reduction methods are proposed. The first one is based on a new companding method that is called Advanced Exponential Companding (AEC) and the second one is a hybrid scheme that combine Zaddoff-Chu Matrix Transform (ZCT) precoding with the proposed AEC companding. Both methods are applied for LTE SISO-OFDM system and LTE MIMOSTBC-OFDM system.

Like standard exponential companding (EC), AEC can effectively transform the original Gaussian-distributed OFDM signals into uniform-distributed (companded) signals without changing the average power level but AEC scheme with same complexity offers better PAPR and BER performance and much design flexibility in the PAPR reduction \& BER performance with $\mathrm{d}$ and $\mathrm{A}$ parameters. Unlike the -law companding scheme, which primarily concentrates on enlarging small signals, our advanced exponential companding modifies both small and large signals without bias so it can display better performance in terms of PAPR reduction and BER. (ZCT + AEC) hybrid scheme combines AEC scheme which transform signal to uniform companded signal with high PAPR \& BER design freedom and constant average power with ZCT precoding technique which has no effects on system BER performance, and does not need power increase, side information and complicated optimization. Combining the advantages of ZCT precoding and AEC $(\mathrm{ZCT}+\mathrm{AEC})$ achieves superior PAPR reduction with great BER improvement and simple implementation. 
The rest of paper is organized as follows: Section 2 explains the PAPR for LTE OFDM system model. Section 3 demonstrates the proposed PAPR reduction schemes in LTE SISO-OFDM and LTE MIMO-OFDM systems. In Section 4, simulation results are presented and discussed. Section 5 concludes the paper.

\section{PAPR of LTE OFDM Systems}

A baseband OFDM system is illustrated in Figure 1, where each OFDM symbol contains $N$ subcarriers. The modulated symbols go through serial to parallel converter block to generate complex vector of size $N$. The vector can be expressed as $X=\left[x_{0}, x_{1}\right.$, $\left.x_{2}, \ldots x_{N-1}\right]^{T}$ and then $X$ goes through the an OFDM modulator block for IFFT transformation, adding cyclic prefix and the time domain complex baseband SISO-OFDM signal with $i=1$ can be written as

$$
X_{n, i}=\frac{1}{\sqrt{N}} \sum_{k=0}^{N-1} X_{k} e^{\frac{j 2 \pi k n}{N}}, 0 \leq n<N
$$

In time domain, the signal $X_{n}$ is really the blended signal of $N$ autonomous subcarriers. Along these lines the SISO-OFDM signal $X n$ at times offers high pinnacles which can be measured by PAPR. The PAPR is defined as the ratio of maximum signal power to average signal power.

$$
\operatorname{PAPR}_{i}=10 \log \frac{\max \left\{\mid X_{n, i}\right\}^{2}}{E\left[\left\{\mid X_{n, i}\right\}^{2}\right]}
$$

In case of MIMO-OFDM system with $i$ transmit antennas, the $P A P R_{M}$ of MIMO-OFDM is actually the maximum PAPR value of all the transmit antennas as:

$$
P A P R_{M}=\max \left(P A P R_{1}, P A P R_{2}, \ldots P A P R_{i}\right)
$$

Commonly, the PAPR performance is measured by the Complimentary Cumulative Distribution Function (CCDF) which is defined as the probability that the PAPR of signal exceeds an assigned threshold. Assuming PAPR0 is the threshold value, CCDF can be expressed as follows,

$$
C C D F=\operatorname{Pr}(P A P R>P A P R 0)
$$

For MIMO-OFDM system, the CCDF of PAPR can be expressed as

$$
C C D F=\left(1-\left(1-e^{-P A P R_{0}}\right)^{i N}\right)
$$

Where $i$ is the number of transmit antennas and $N$ is the number of subcarriers with each antenna.

\section{Proposed PAPR Reduction Method}

The proposed LTE SISO-OFDM and LTE MIMO-OFDM systems with AEC and (ZCT + AEC) hybrid PAPR reduction are shown in Figure 2 and Figure 3 respectively. We refer to the modulated signal vector as $X=[X 0, X 1, X 2, \ldots X N-1]^{T}$ converted from serial to parallel by $\mathrm{p} / \mathrm{s}$ block, then processed by ZCT precoding transform block, and its output $z$ can be written as

$$
z=Z \times X
$$

where $Z$ is the $N \times N Z C T$-transform precoding matrix, the output signal $z=\left[z_{0}, z_{1}, z_{2}, \ldots z_{N-1}\right]^{T}$ passing through OFDM Modulator block to perform Inverse Fast Fourier Transform (IFFT), and add cyclic prefix and can be written as

$$
x=\operatorname{IFFT}(z)
$$

the output signal $x=\left[x_{0}, x_{1}, x_{2}, \ldots x_{N-1}\right]^{T}$ passing through advanced exponential companding and can be written as 


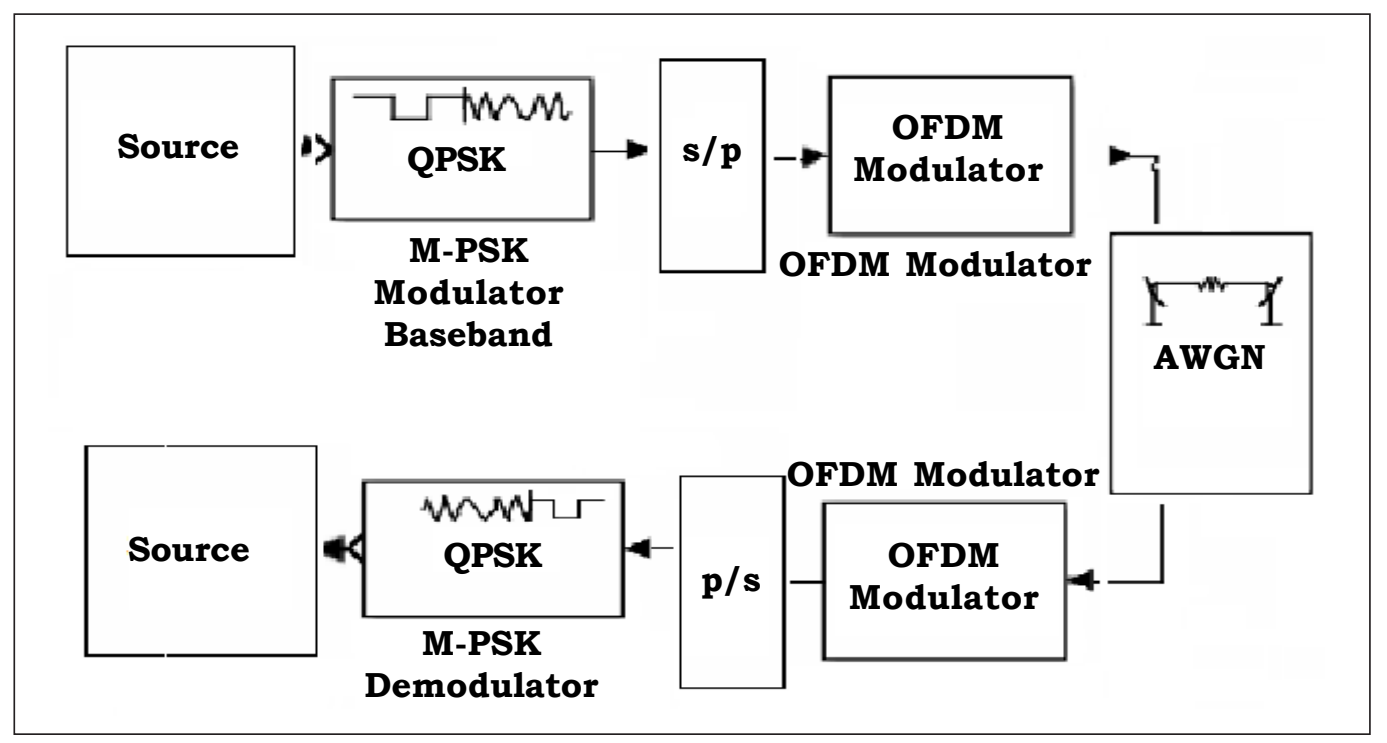

Figure 1. Block diagram for LTE SISO-OFDM system model

$$
y=A(x)
$$

where $A(\cdot)$ denotes the AEC function and $y$ is the signal to be sent into wireless channels. Assuming $h$ is the channel impulse response and $w$ is the additive white Gaussian noise, the received signal can be expressed as

$$
R x=y * h+w
$$

With the de-companding operation, the recovered signal can be expressed as

$$
D e=A^{-1}(R x)
$$

Then De is fed into the OFDM Demodulator block for removing CP, applying FFT transform and the signal after this transform is

$$
Y=F F T(D e)
$$

The output signal of OFDM Demodulator $Y$ enters inverse ZCT block and the out signal of this block is

$$
\text { Zout }=Z^{-1} \times Y
$$

where $Z^{-1}$ is $N \times N$ inverse of ZCT matrix precoding then the signal Zout is demodulated producing the oringinal data. The proposed scheme includes two stages of PAPR reduction. One is ZCT precoding and the other is the advanced exponential companding both of them explained with the following subsections.

Like LTE SISO-OFDM system, LTE MIMO-OFDM signal has similar behavior except that ZCT precoding and AEC scheme hav been applied with all antennas after STBC coding as shown in Figure 3.

\subsection{Advanced Exponential Companding (AEC)}

This part offers mathematical expressions of AEC technique. It has the following equation at the transmission side:

$$
h(x)=\operatorname{sgn}(x) \sqrt[d]{\alpha\left[1-A^{\left(-\frac{|x|^{2}}{\sigma^{2}}\right)}\right]}
$$

Where $\operatorname{sgn}(x)$ is sign function, $A$ represents constant which change from 2.7 to 100 and the positive constant $\alpha$ determines the 
average power output signals. In order to keep the input and output signals at the same average power level

$$
\alpha=\left[\frac{E\left[|x|^{2}\right]}{E\left[\sqrt[d]{\left[1-A^{\left(-\frac{|x|^{2}}{\sigma^{2}}\right)}\right]}\right]^{\frac{d}{2}}}\right.
$$

At the receiver side, the inverse function of $h(x)$ is used in the decompanding operation:

$$
h^{-1}(x)=\left|\sqrt{-\sigma^{2} \frac{\log _{e}\left(1-\frac{|x|^{d}}{\alpha}\right)}{\log _{e}(A)}}\right|
$$

\subsection{Zadoff-Chu Matrix Transform Precoding (ZCT)}

ZCT Precoding based OFDM system extracted from Zadoff-Chu sequences which are type of poly phase sequences that have optimum correlation properties. Zadoff-Chu sequences have an ideal periodic autocorrelation and constant magnitude. The Zadoff-Chu sequences of length $L$ can be defined:

$$
z(k)=\left[\begin{array}{ll}
e^{\left(\frac{j 2 \pi r\left(k^{2}+2 q k\right)}{2 L}\right)} & \text { for L even } \\
e^{\left(\frac{j 2 \pi r(k(k+1)+2 q k)}{2 L}\right)} \text { for L odd }
\end{array}\right]
$$

where $k=0,1,2 \ldots L-1, q$ is any integer, $r$ is any integer relatively prime to $L$. With the use of reordering and letting, $q=1$ and $r$ $=1$ and substitute $k=m N+1$ in equation 16 for $L$ Even we get $R$ matrix which is a ZCT based row-wise precoding matrix of size $L=(N \mathrm{X} N)$ and can be written as:

$$
R=\left[\begin{array}{llll}
r_{00} & r_{01} & \ldots & r_{0(N-1)} \\
r_{10} & r_{11} & \ldots & r_{1(N-1)} \\
\cdot & \cdot & \cdot & \cdot \\
\cdot & \cdot & \cdot & \cdot \\
r_{(N-1) 0} & r_{(N-1) 1} & \ldots & r_{(N-1)(N-1)}
\end{array}\right]
$$

In the ZCT precoding based OFDM system baseband modulated information is gone through $\mathrm{S} / \mathrm{P}$ block which creates a complex vector of size $N$ that can be typed as $X=[X 0, X 1, X 2, \ldots X N-1]^{T}$. Then ZCT precoding is applied to this complex vector and converts this complex vector into new vector of length $N$ that can be written as $Y=R X=[Y 0, Y 1, Y 2 \ldots Y N-1]^{T}$.

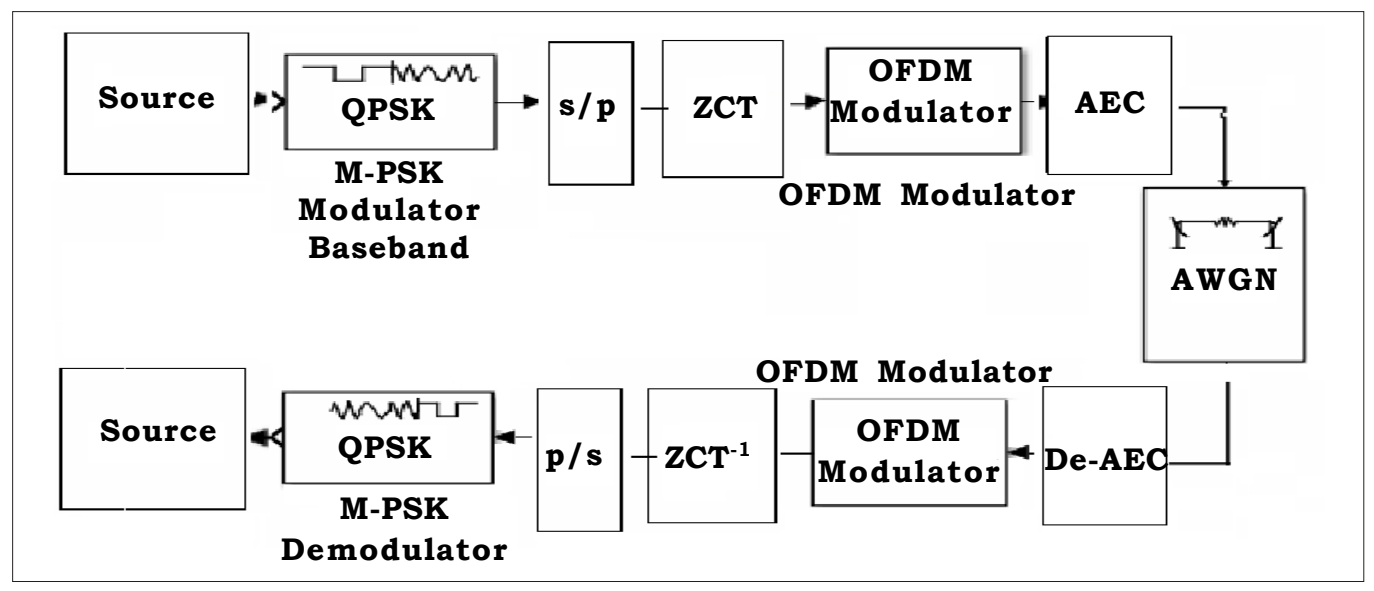

Figure 2. Hybrid proposed PAPR reduction method for LTE SISO-OFDM 


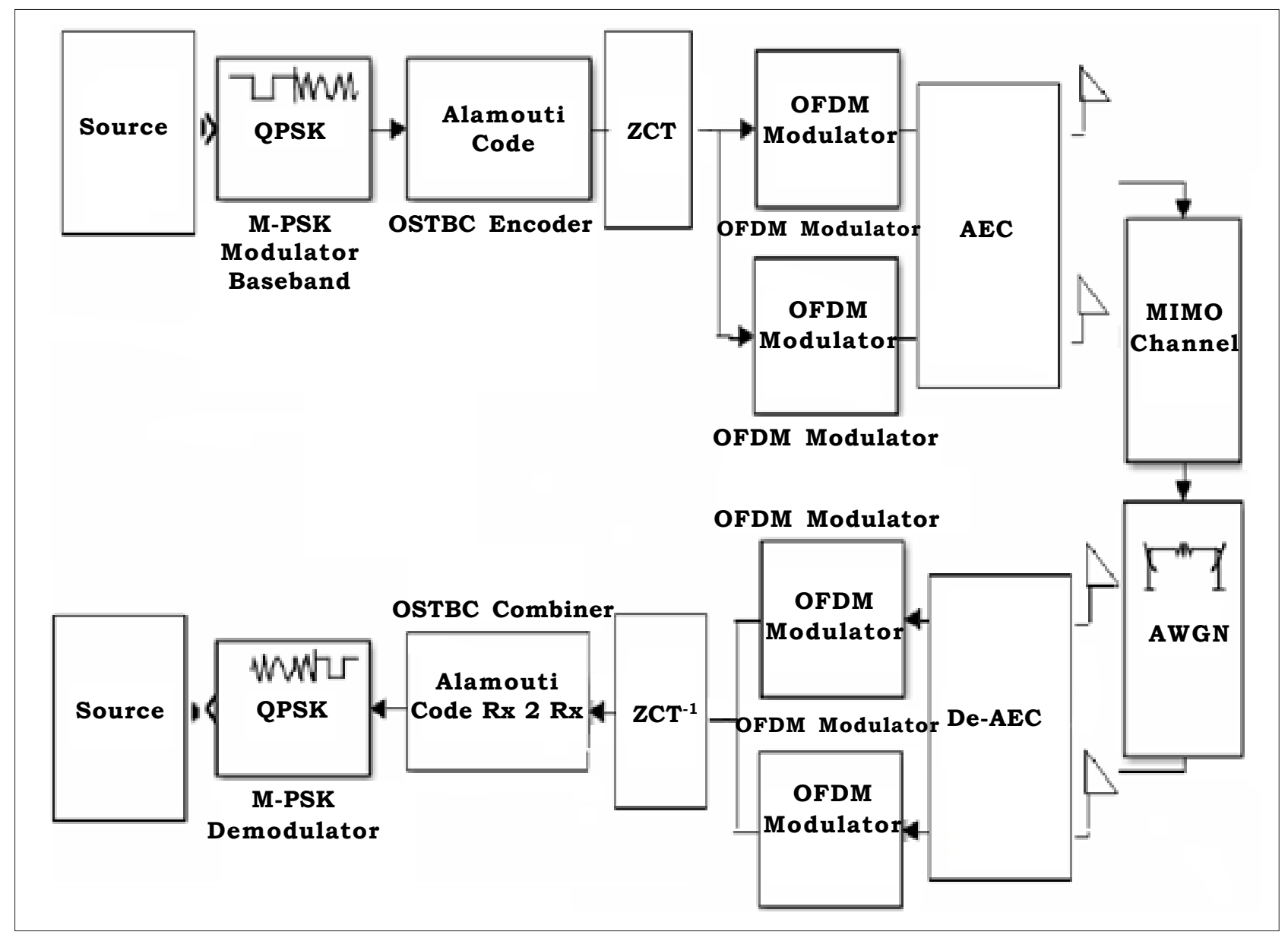

Figure 3. Hybrid proposed PAPR reduction method for LTE MIMO-OFDM system

\section{Simulation Results}

\subsection{LTE SISO-OFDM}

To evaluate the performance of the proposed scheme, simulation results are presented in this section. In all simulations, QPSK modulation is adopted, the number of subcarrier $N$ has been set to 128, the oversampling factor $L=4$ and the length of cyclic prefix is chosen to be 32. AWGN channels are applied in all simulations. With the consideration of system performance, the parameter $\mathrm{d}$ of AEC is set to 1 .

In several sets of simulations, we compare the proposed schemes of (AEC) and hybrid (AEC + ZCT) with traditional LTE SISOOFDM. Besides that, to show the effect of AEC on system performance, we make a comparison among AEC, standard exponential companding (EC) and Mu-law companding. Also, to see the effect of hybrid (ZCT + AEC) on system performance, we make a comparison among (ZCT + AEC), (ZCT + Mu-law), (DCT + Mu-law), (DHT + Mu-law), (WHT + Mu-law), and (WHT + EC) hybrid techniques. The system performance of proposed advanced exponential companding (AEC) method in terms of PAPR \& BER is shown in Figure 4 and Figure 5 respectively, we note that the PAPR performance decreases while BER performance increases when A parameter increases. At $A=100$ the best PAPR performance of $1.6 \mathrm{~dB}$ achieved at the cost of $9 \mathrm{~dB}$ worst SNR loss with respect to traditional LTE SISO-OFDM.

Figure 6 and Figure 7 show the PAPR and BER performance for advanced exponential companding (AEC) compared with existing schemes of Mu-Law companding and standard exponential companding (EC). By comparison with standard exponential companding (EC), we note that our proposed scheme (AEC) at $A=2.7$ has the same PAPR value of $5.16 \mathrm{~dB}$ as (EC) scheme at $d=1$ but our scheme has $4.5 \mathrm{~dB}$ SNR less (better) than 5.5dB SNR for (EC) scheme at the same level. As seen our scheme (AEC) 


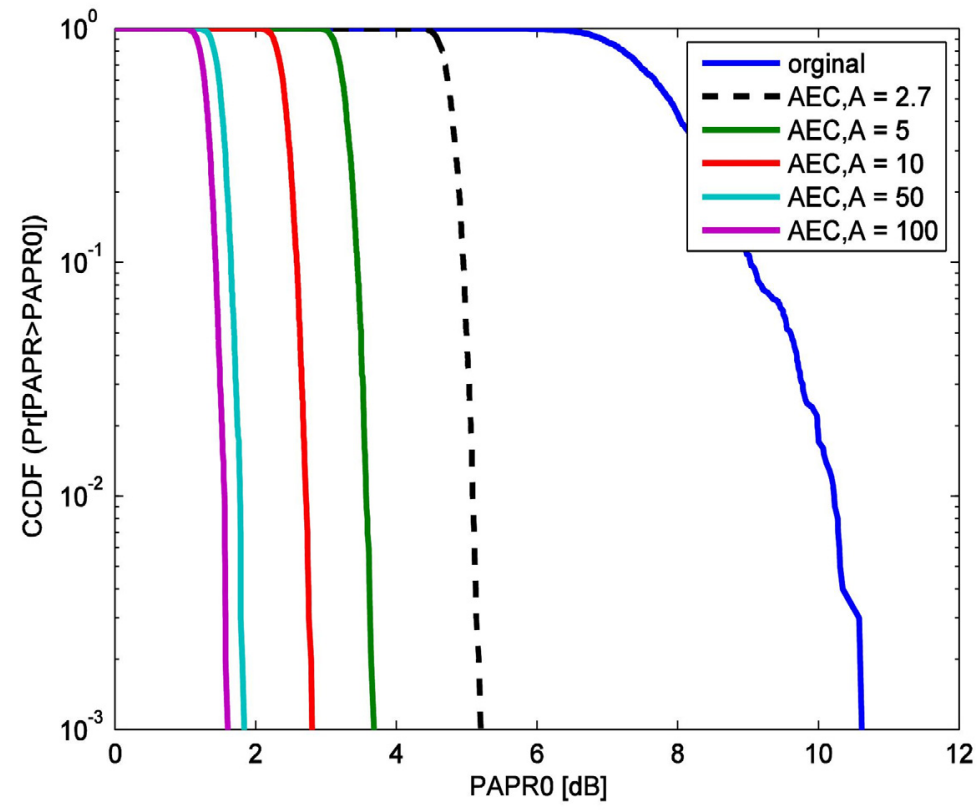

Figure 4. PAPR performance for proposed advanced exponential companding (AEC)with LTE SISO-OFDM system

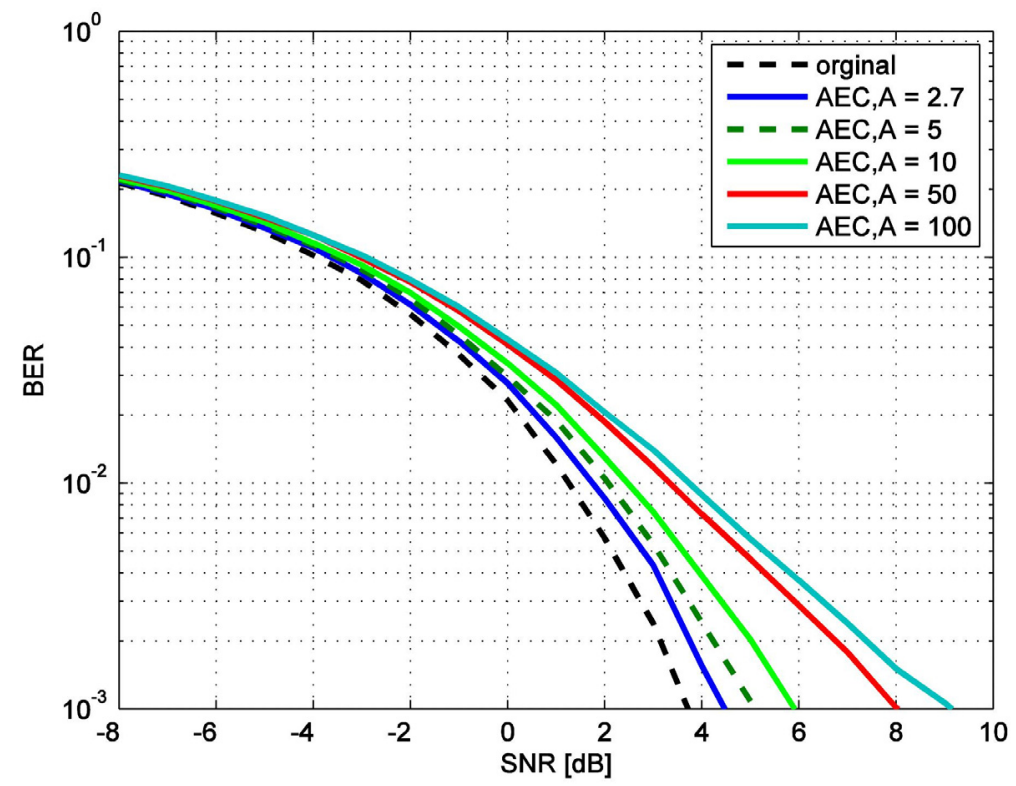

Figure 5. BER performance for proposed advanced exponential companding (AEC) with LTE SISO-OFDM system

with parameter $A \geq 10$ until A=100 achieves better PAPR \& BER performance than (EC) scheme at $d=2$ where the best PAPR \& SNR improvements of our scheme are $1.6 \& 0.9 \mathrm{~dB}$ at $A=100$ against (EC) scheme at $d=2$. By comparison with Mu-Law companding, we adjust $\mu$ parameter to 14 and 500 and see that our proposed scheme (AEC) at $A=50$ and 100 achieves better PAPR \& BER performance than Mu-Law companding at $\mu=500$. Also, our scheme (AEC) at $A=2.7$ reduces PAPR to $5.1 \mathrm{~dB}$ with increase in SNR at $10^{\wedge}-3$ BER to $4.3 \mathrm{~dB}$ with respect to traditional LTE SISO-OFDM signal while Mu-Law companding decreases PAPR to $5.1 \mathrm{~dB}$ with increase in SNR at $10^{\wedge}-3$ BER to $5.9 \mathrm{~dB}$ at $\mu=14$. This means that our scheme (AEC) at $A=2.7$ provides the same PAPR performance with (5.9-4.3) 1.6dB SNR improvement (gain) with respect to Mu-Law companding at parameter $\mu=14$. From pervious we say that AEC proposed method offers high PAPR \& BER performance than standard exponential companding (EC) and Mu-Law companding. 


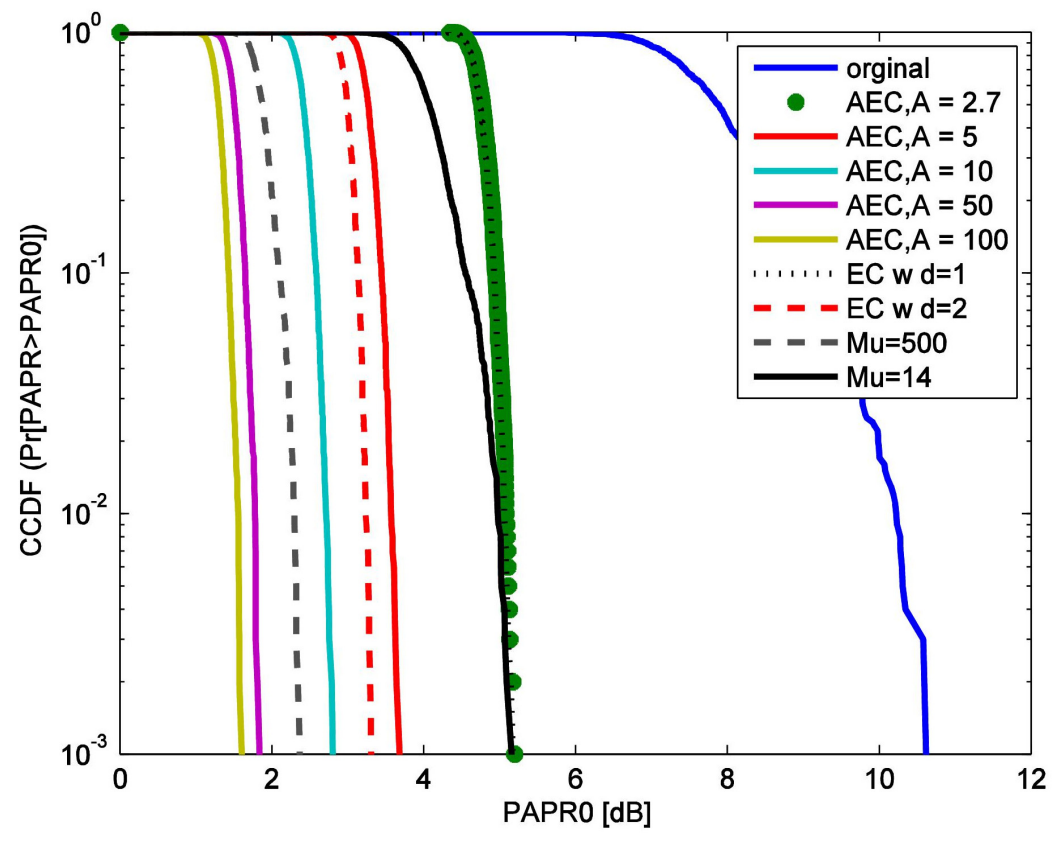

Figure 6. PAPR performance for advanced exponential companding (AEC) with different A values compared to standard existing exponential companding and Mu-law companding of LTE SISO-OFDM system

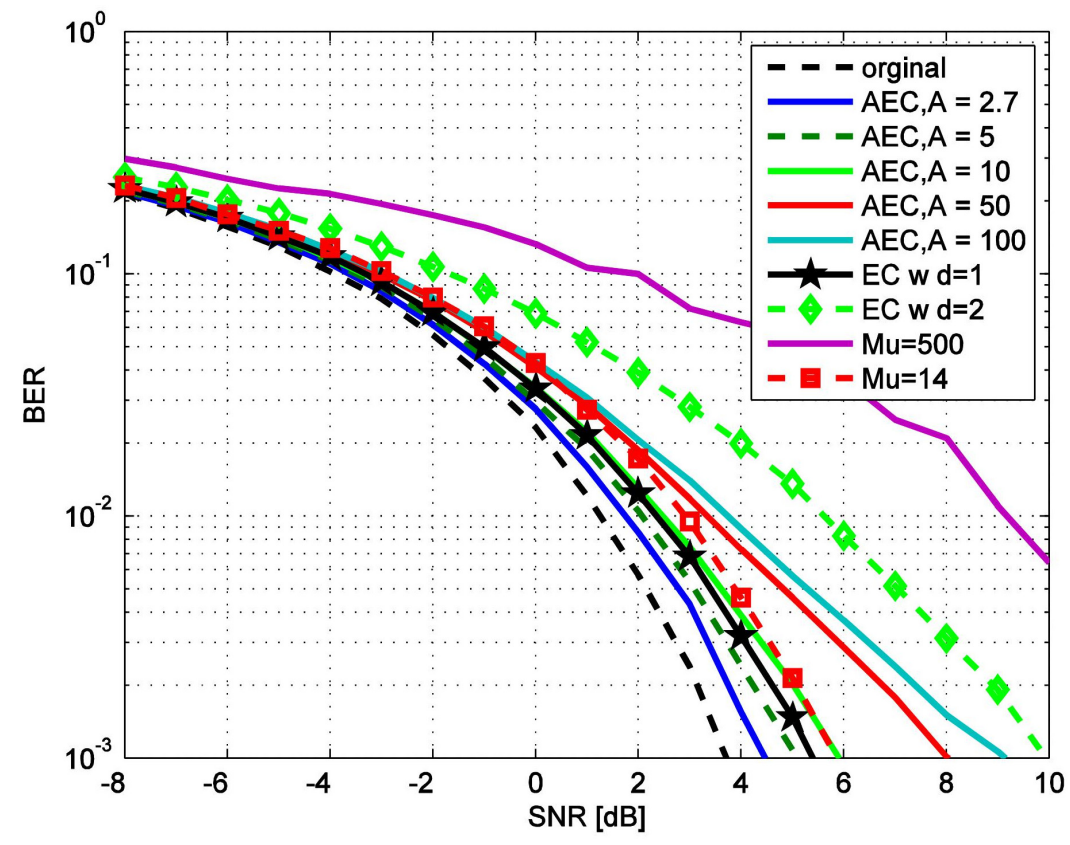

Figure.7. BER performance for advanced exponential companding (AEC) with different A values compared to standard existing exponential companding and Mu-law companding of LTE SISO-OFDM system

Figure 8 and Figure 9 present PAPR and BER performance of proposed (ZCT + AEC) hybrid scheme compared with conventional LTESISO-OFDM signal where PAPR decreases while SNR increases when A value increases, the best PAPR result is $0.9 \mathrm{~dB}$ with 5.6dB worst SNR at $A=100$. By comparison this hybrid scheme with AEC scheme in Figure 4 and Figure 5, we observe that proposed $(\mathrm{ZCT}+\mathrm{AEC})$ hybrid scheme presents both high PAPR \& BER performance than AEC scheme overall A parameters and the best improvements of PAPR \& BER at $\mathrm{A}=100$ are (1.6-0.9) $0.7 \&(9-5.6) 3.4 \mathrm{~dB}$ respectively. 


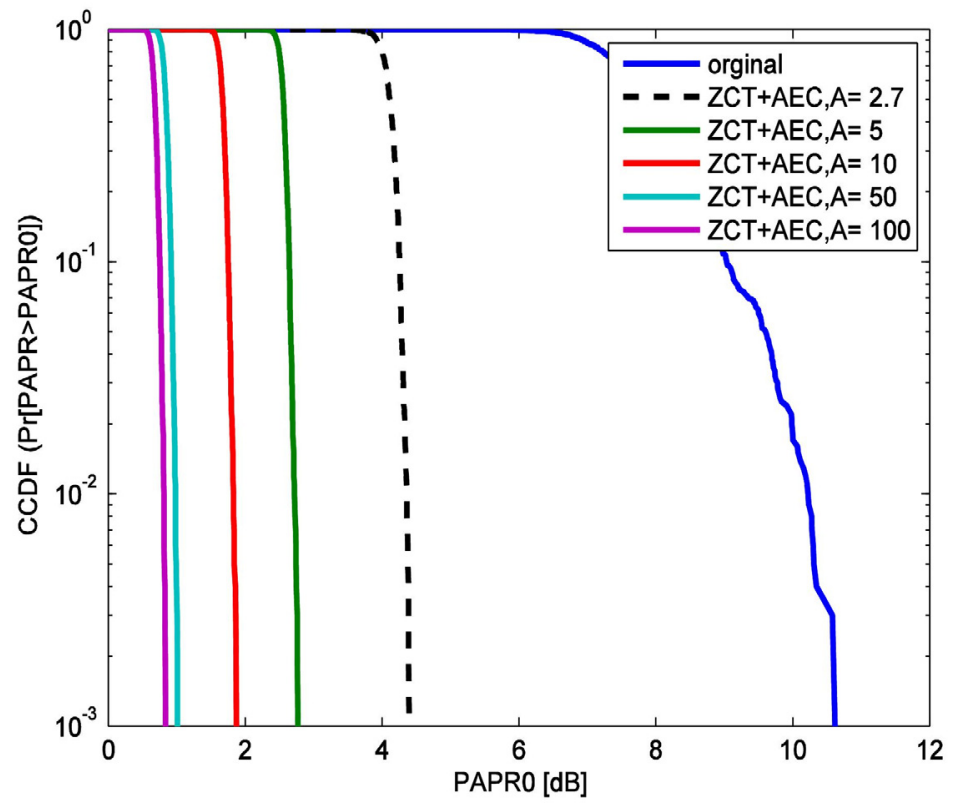

Figure 8. PAPR performance for proposed hybrid scheme using ZCT precoding and advanced exponential companding (AEC) with different A values of LTE SISO-OFDM system

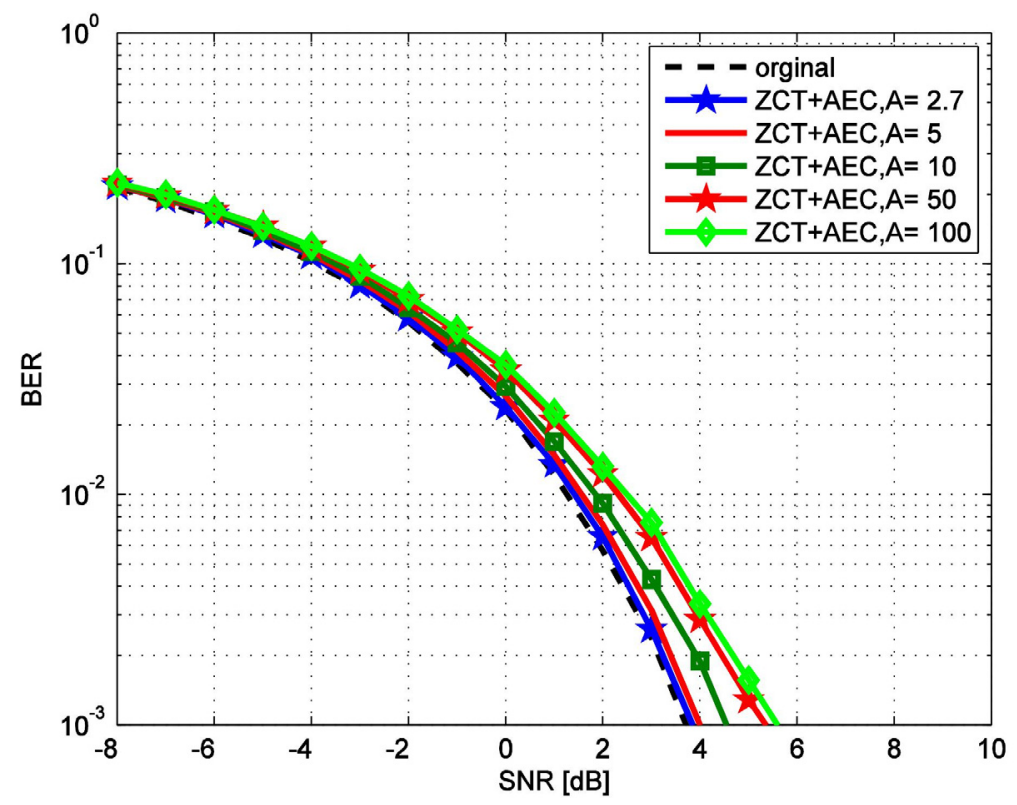

Figure 9. BER performance for proposed hybrid scheme using ZCT precoding and advanced exponential companding (AEC) with different A values of LTE SISO-OFDM system

Finally, Figure 10 and Figure 11 offer PAPR and BER performance for proposed (ZCT + AEC) hybrid scheme compared with current related hybrid schemes. It is clear that the proposed (ZCT + AEC) hybrid scheme achieves the least PAPR \& BER performance among others schemes where our hybrid proposed scheme (ZCT + AEC), (ZCT + Mu-Law), (DCT + Mu-Law), (DHT + Mu-Law), (WHT + Mu-Law), and (WHT + EC) schemes reduce the PAPR value to 1.9, 2.1, 2.2, 2.9, 3.2, and 5dB but increase SNR to 4.5, 5.2, 8, 8, 9.5, and 9.5dB respectively compared to traditional LTESISO-OFDM. We conclude that our proposed hybrid sheme $(\mathrm{ZCT}+\mathrm{AEC})$ provides better improvements in PAPR \& BER performance against alone AEC scheme, $(\mathrm{ZCT}+\mathrm{Mu}-\mathrm{Law})$, (DCT + Mu-Law), (DHT + Mu-Law), (WHT + Mu-Law), and (WHT + EC) schemes. 


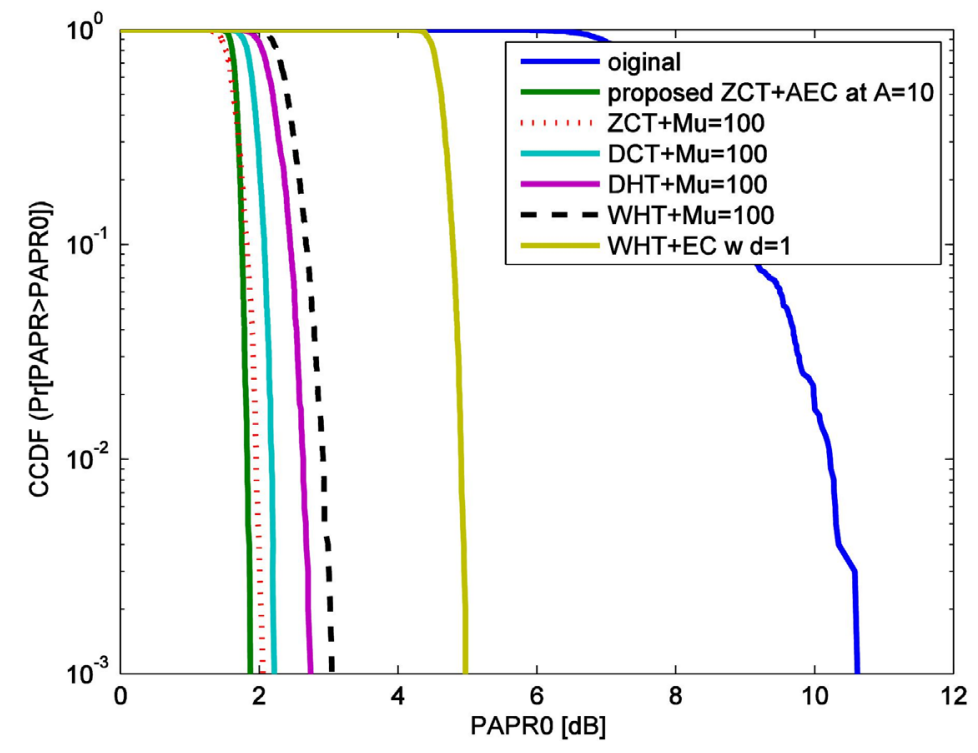

Figure 10. PAPR performance for proposed hybrid scheme using ZCT precoding and advanced exponential companding (AEC) with different A values compared to existing related schemes of LTE SISO-OFDM system

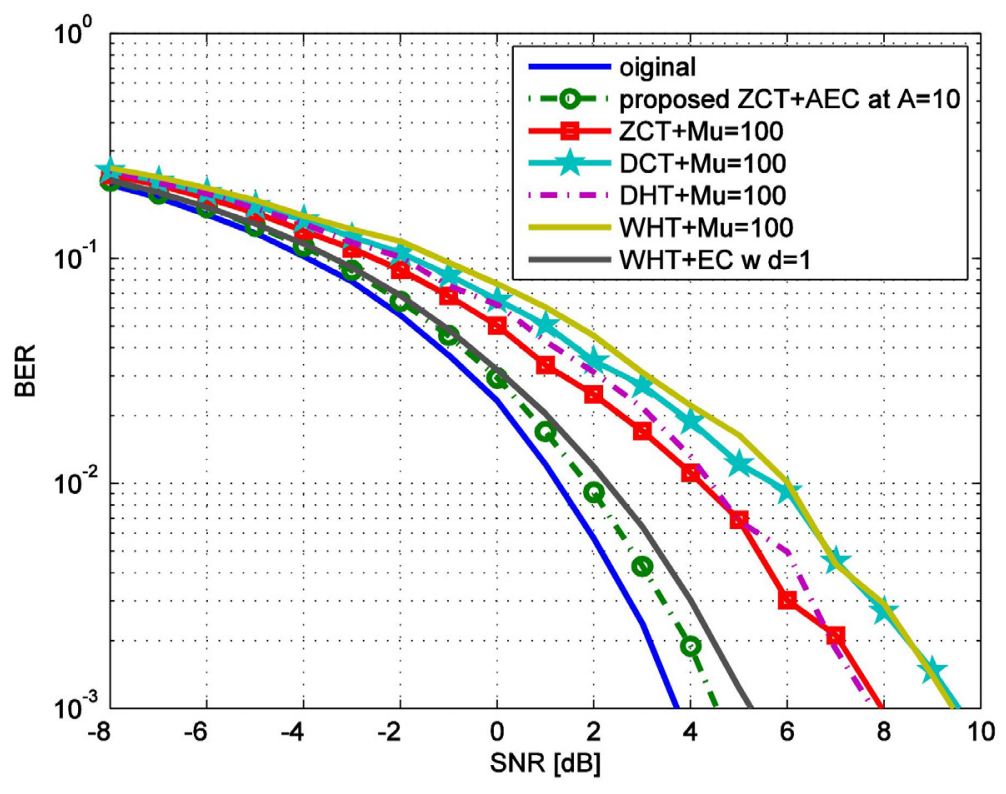

Figure 11. BER performance for proposed hybrid scheme using ZCT precoding and advanced exponential companding (AEC) with different A values compared to existing related schemes of LTE SISO-OFDM system

\subsection{LTE MIMO-OFDM}

To evaluate the performance of the proposed schemes with LTE MIMO-OFDM system (STBC $2 * 2$ ), simulation results are presented in this section. In simulations, QPSK modulation is adopted, the number of subcarrier $N=128$, the oversampling factor $L=4$, the number of transmit antennas $2 \mathrm{Tx}$, the number of receive antennas $2 \mathrm{Rx}$, and the length of cyclic prefix is 32 . AWGN and flat fading MIMO channels are used.

In simulations, we compare the proposed schemes of (AEC) and hybrid (ZCT + AEC) with traditional LTE MIMO-OFDM. Besides that, to view the effect of $(\mathrm{ZCT}+\mathrm{AEC})$ on system performance, we make a comparison among (ZCT + AEC), existing ZCT precoding, and AEC scheme. 
Figure 12 and Figure 13 demonstrate PAPR and BER performance of proposed advanced exponential companding (AEC) respectively with LTE MIMO-OFDM system. It is obvious that AEC scheme offers better PAPR improvement (large) at the cost of SNR loss (increase) when A values increases with respect to traditional LTE MIMO-OFDM. The AEC scheme with best result reduces the PAPR to $1.6 \mathrm{~dB}$ at the expense of worst increase in SNR to $5.9 \mathrm{~dB}$ at $A=100$. We note that the PAPR of traditional LTE MIMO-OFDM is $11.1 \mathrm{~dB}$ while the PAPR of traditional LTE SISO-OFDM is $10.5 \mathrm{~dB}$ because we transmit two OFDM time slots with two antennas of LTE MIMO-OFDM system $(2 * 2)$ instead of one OFDM time slot with LTE SISO-OFDM system so the PAPR improvement (gain) obtained by AEC scheme with LTE MIMO-OFDM system (2*2) is better (larger) than PAPR improvement obtained by AEC scheme with LTE SISO-OFDM system. Also, the SNR at 10^-3 BER improvement (gain) obtained by AEC scheme with LTE MIMO-OFDM system (2*2) is better (less) than SNR improvement obtained by AEC scheme with LTE SISOOFDM system.

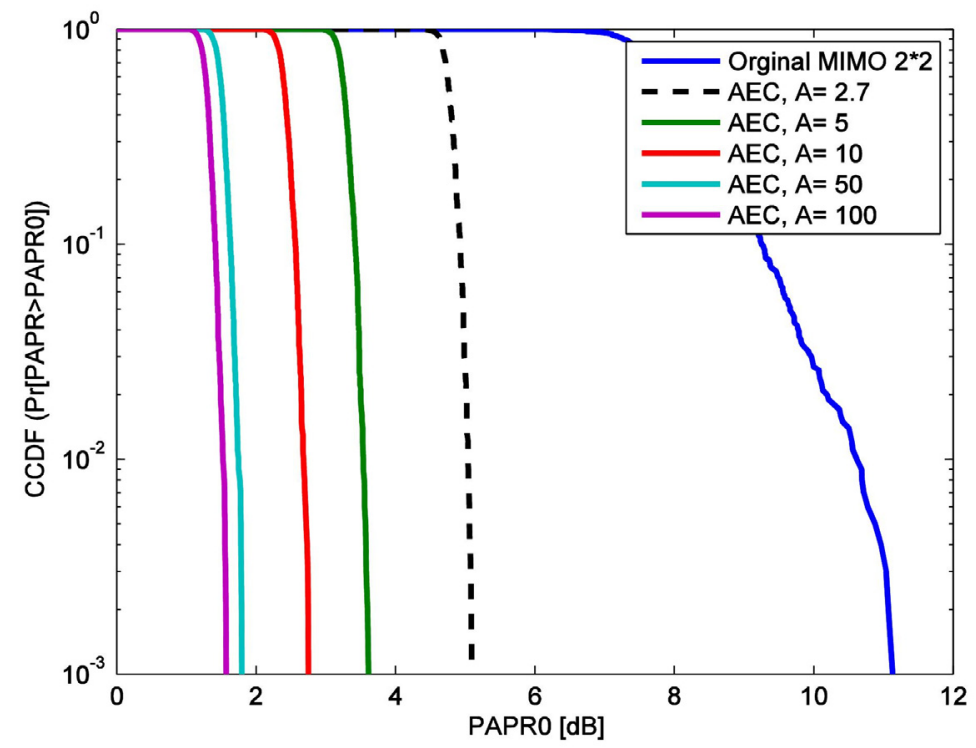

Figure 12. PAPR performance for advanced exponential companding (AEC) proposed scheme with different A values of LTE MIMO-OFDM system

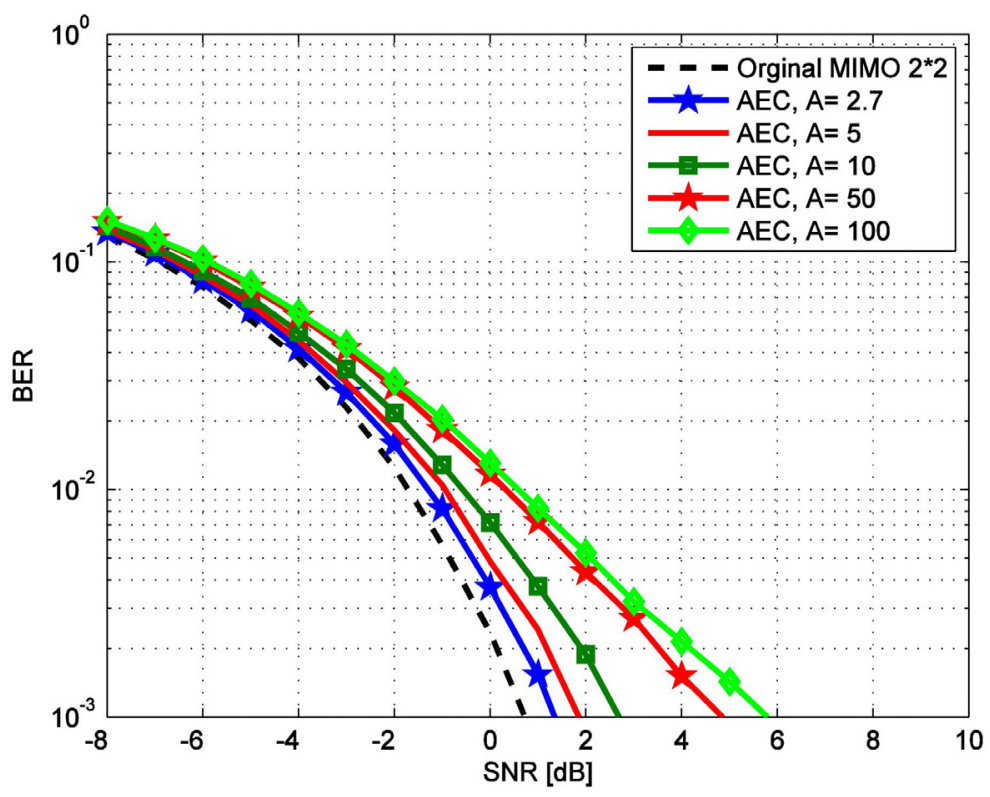

Figure 13. BER performance for advanced exponential companding (AEC) proposed scheme with different A values of LTE MIMO-OFDM system 
Figure 14 and Figure 15 show the PAPR and BER performance for existing ZCT precoding scheme with LTE MIMO-OFDM system $(2 * 2)$ where ZCT precoding scheme reduces the PAPR to $7.5 \mathrm{~dB}$ with keeping the same SNR at $10^{\wedge}-3$ BER of traditional LTE MIMO-OFDM.

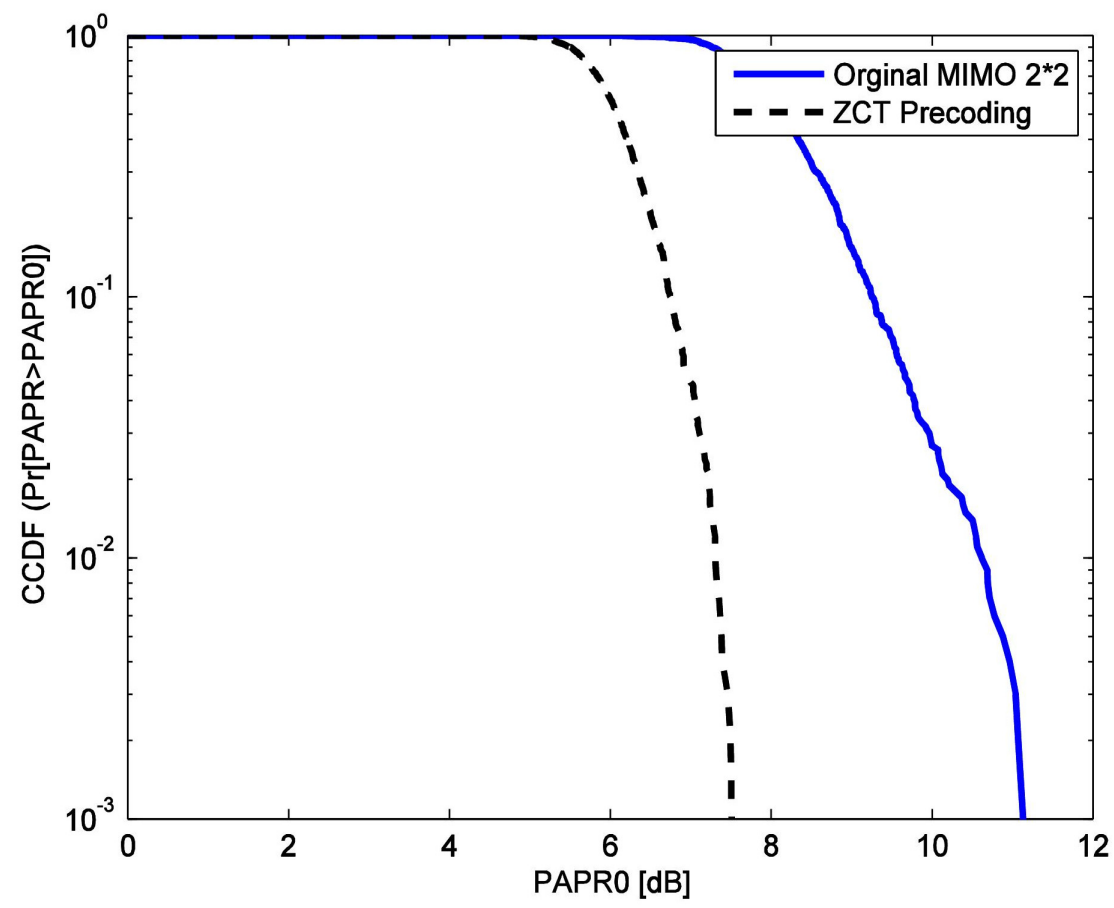

Figure 14. PAPR performance for existing ZCT precoding scheme of LTE MIMO-OFDM system

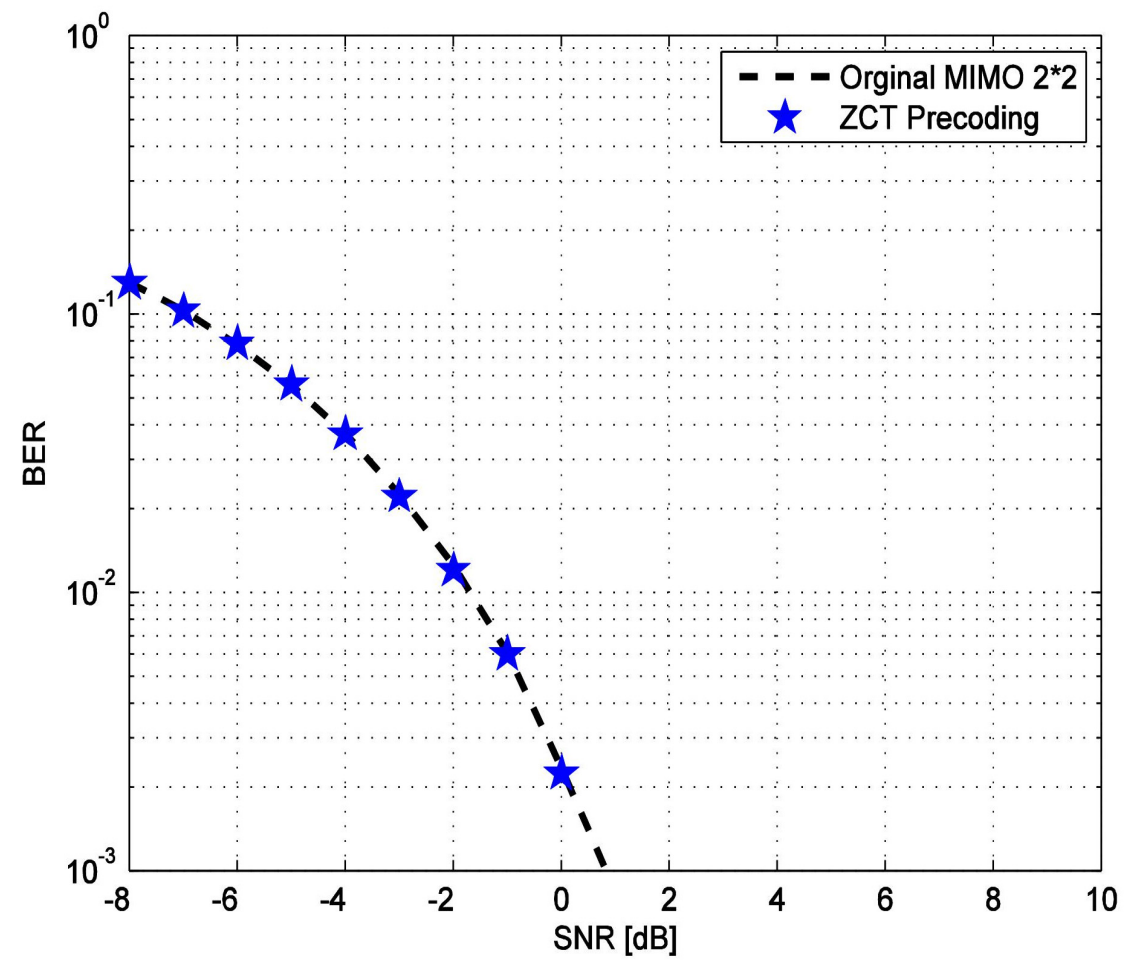

Figure 15. BER performance for existing ZCT precoding scheme of LTE MIMO-OFDM system 
Figure 16 and Figure 17 present the PAPR and BER performance for our proposed (ZCT + AEC) hybrid method with LTE MIMOOFDM system $(2 * 2)$ where (ZCT + AEC) hybrid method improves the PAPR (decreases) at the expense of slight deterioration in SNR at $10^{\wedge}-3$ BER with respect to traditional LTE MIMO-OFDM performance. By comparison this hybrid scheme to AEC scheme in Figure 12 and Figure 13, We see that the (ZCT + AEC) hybrid method provides more PAPR reduction with better (less) SNR at $10^{\wedge}-3$ BER performance than AEC scheme where (ZCT + AEC) hybrid method with best result at $A=100$ reduces the PAPR to $0.9 \mathrm{~dB}$ at the cost of SNR increase only to $2 \mathrm{~dB}$ while AEC method at $A=100$ reduces the PAPR to $1.6 \mathrm{~dB}$ at the cost of SNR increase to $5.9 \mathrm{~dB}$. And finally proposed (ZCT + AEC) hybrid method presents high PAPR performance at the expense of slight degradation in SNR at $10^{\wedge}-3$ BER performance against existing ZCT precoding scheme in Figure 13 and Figure 16 with more design flexibility.

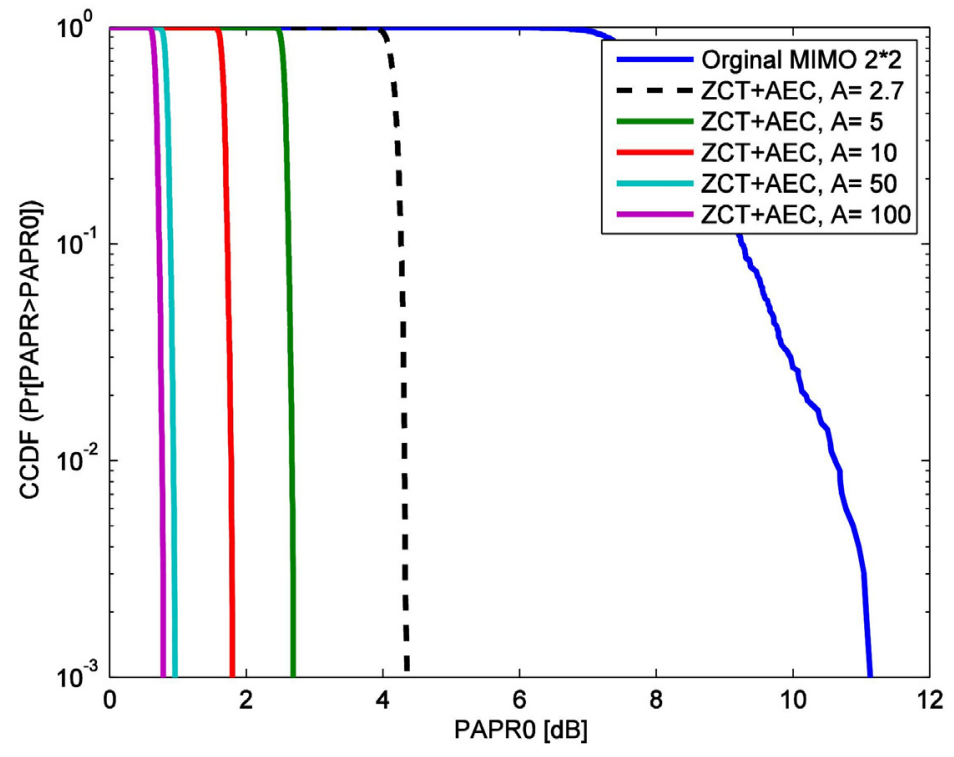

Figure 16. PAPR performance for proposed hybrid scheme using ZCT precoding and advanced exponential companding (AEC) with different A values compared to existing related schemes of LTE MIMO-OFDM system

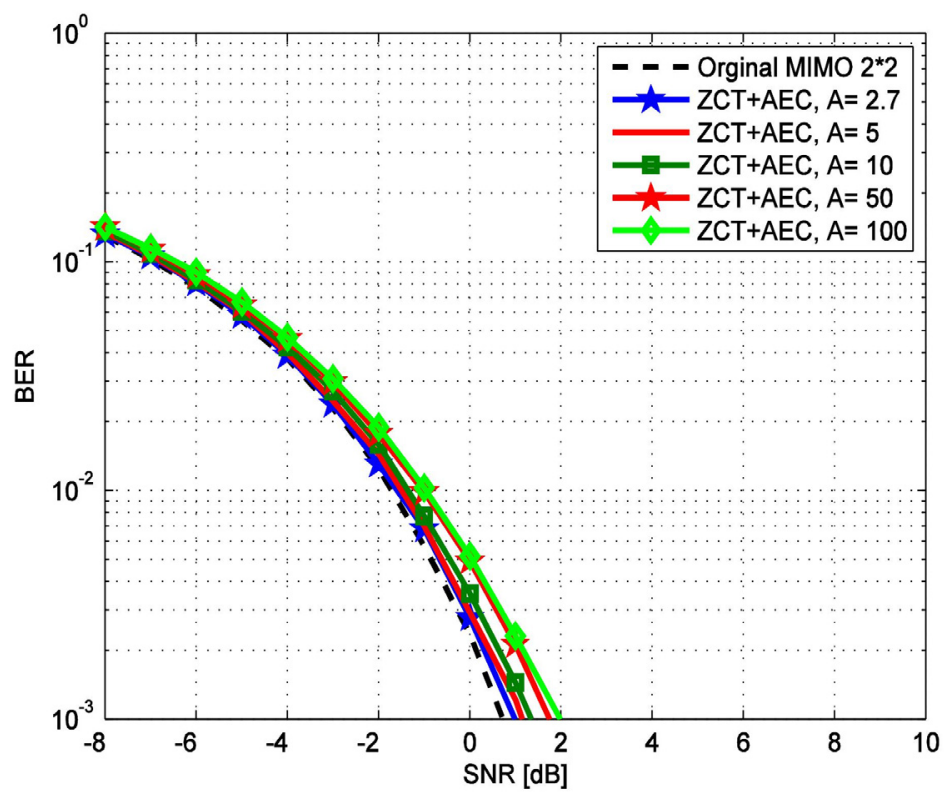

Figure 17. BER performance for proposed hybrid scheme using ZCT precoding and advanced exponential companding (AEC) with different A values compared to existing related schemes of LTE MIMO-OFDM system 


\section{Conclusion}

In this paper, we have suggested new PAPR techniques; advanced exponential commanding (AEC) and a hybrid system which includes Zaddoff Chu Matrix Transform (ZCT) precoding with AEC (ZCT + AEC) both are applied to LTE SISO-OFDM and LTE MIMO-OFDM systems. AEC with LTE SISO-OFDM system can achieve more reduction in PAPR and significant improvement in BER with same complexity, same average power and provides much more design flexibility in the PAPR reduction \& BER performance against standard exponential companding (EC) and Mu-Law companding. Moreover, AEC with LTE MIMO-OFDM system offers better PAPR improvements with larger improvement in BER with those of LTE SISO-OFDM system. (ZCT + AEC) hybrid technique with both LTE SISO-OFDM system and LTE MIMO-OFDM system presents superior improvement in both PAPR and BER performance against alone AEC method. In addition, (ZCT + AEC) hybrid technique with LTE SISO-OFDM system achieves higher improvement in both PAPR and BER performance with no power increment, no side information and more design flexibility with respect to those existing hybrid methods; (discrete hartley transform (DHT) precoding + Mu-law companding) [28], (Zadoff -chu matrix transform (ZCT) + Mu-law companding) [29], (Walsh Hadamard Transform (WHT) precoding + Mu-law companding) [29], (Walsh Hadamard Transform (WHT) precoding + exponential companding (EC)) [30], and (discrete cosine transform (DCT) precoding + Mu-law companding) [25], but (ZCT + AEC) hybrid technique with LTE MIMO-OFDM system greatly enhances the PAPR performance at the expense of slight loss in BER performance against current ZCT precoding scheme.

The hybrid scheme we have assumed is applied more practical with MIMO systems, collects the advantages and offsets the deficiency of the two techniques and outperforms existing methods.

\section{References}

[1] Shu, S., Qu, D., Li, L., Jiang, T. (2015). Invertible subset QC-LDPC codes for PAPR reduction of OFDM signals, IEEE Transactions on Broadcasting, 61, 290-298.

[2] Naidu, G., Rao, D. S., Prasad, D. (2015). Improved performance of OFDM systems with reduced PAPR based on channel coding techniques, In: Electrical, Electronics, Signals, Communication and Optimization (EESCO), 2015 International Conference on, 2015, 1-6.

[3] Shiragapur, B. K., Wali, U. (2016). Peak-to-Average Power ratio reduction using coding and hybrid techniques for OFDM system, ICTACT Journal on Communication Technology, vol. 7.

[4] Bouaziz, S., Rekkal, K. (2015). A PAPR reduction for STBC MIMO-OFDM systems in 4G wireless communications using PTS scheme, In: New Technologies of Information and Communication (NTIC), 2015 First International Conference on, 2015, 1-5.

[5] Vittal, M., Naidu, K. R. (2017). A novel reduced complexity optimized PTS technique for PAPR reduction in wireless OFDM systems, Egyptian Informatics Journal, 18, 123-131.

[6] Das, J., Bansode, R. (2016). PAPR Reduction using PTS-PSO technique for 16× 16 MIMO-OFDM Systems with 16-QAM, Global Journal of Computer Science and Technology, vol. 15.

[7] Patil, P. S., Kota, P., Gaikwad, A. (2016). Study of PTS based technique to reduce PAPR in SFBC MIMO OFDM system with reduced complexity, International Journal, vol. 5.

[8] Hung, H.-L., Huang, Y.-F., Wei, C.-C., Chen, R.-C. (2016). Performance of PTS-Based Firefly Algorithm Scheme for PAPR Reduction in SFBC MIMO-OFDM Communication Systems, In: 2016 International Symposium on Computer, Consumer and Control (IS3C), 2016, 854-857.

[9] Rangaswamy, M., Ravikumar, A., Gayathri, K., Yamuna, K. (2016). An Effective Approach for Peak Average Power Ratio Reduction in Multi input Multioutput OFDM systems.

[10] Haque, S. S., Mowla, M. M., Hasan, M. M., Bain, S. K. (2015). An algorithm for PAPR reduction by SLM technique in OFDM with hadamard matrix row factor, In: Electrical Engineering and Information Communication Technology (ICEEICT), 2015 International Conference on, 2015, 1-7.

[11] Taspinar, N., Yildir1m, M. (2015). A Novel Parallel Artificial Bee Colony Algorithm and Its PAPR Reduction Performance Using SLM Scheme in OFDM and MIMO-OFDM Systems, IEEE Communications Letters, 19, 1830-1833.

[12] Sohn, I. (2016). New SLM scheme to reduce the PAPR of OFDM signals using a genetic algorithm, ICT Express, 2, 63-66. 
[13] Tören, M., Çiflikli, C. (2016). Comparison of Reduction Methods for Peak-to-Average-Power-Ratio (PAPR) in MIMO-OFDM Systems with a New Approach, Acta Physica Polonica A, 130, 417-421.

[14] Poudel, B., Mishra, B. (2016). Performance Analysis of PAPR Reduction in $4 \times 4$ Spatially Multiplexed MIMO-OFDM System using SLM and Optimum-PTS Techniques, Journal of Telecommunications System and Management, 5, 2167-0919.100012, 2016.

[15] Gamage, H., Rajatheva, N., Latva-aho, M. (2016). High PAPR Sequence Scrambling for Reducing OFDM Peak-to-Average Power Ratio, in European Wireless 2016; 22th European Wireless Conference, 2016, 1-5.

[16] Chakraborty, M. K. (2015). Reduction of Peak Power Average Ratio in OFDM System Using Clipping.

[17] Singh, S., Kumar, A. (2016). Performance Analysis of Adaptive Clipping Technique for Reduction of PAPR in Alamouti Coded MIMO-OFDM Systems, Procedia Computer Science, 93, 609-616.

[18] Vangala, S., Sundru, A. (2016). Adaptive Clipping Active Constellation Extension for PAPR Reduction of OFDM/OQAM System, Procedia Computer Science, 93, 617-623.

[19] Kota, P., Gaikwad, A., Patil, B. (2015). BER analysis of PAPR reduction techniques in SFBC OFDM system: A review, in 2015 International Conference on Information Processing (ICIP), 2015, 108-113.

[20] Shafter, E., Rao, R. K. (2016). A Comparison between SLM, PTS, and CF Schemes for the Reduction of PAPR of OFDM System with CPM Mappers, IAENG International Journal of Computer Science, 43.

[21] Jamadar, M. N., Patil, S. (2015). Performance Improvement in MIMO-OFDM System by Papr Reduction, International Journal for Innovative Research in Science and Technology, 2, 14-22.

[22] Ramaswamy, T., Reddy, K. C. (2016). Low Complexity Iterative Piece-Wise Companding Transform for Reduction of PAPR in MIMO OFDM Systems, Indian Journal of Science and Technology, 9.

[23] Das, M., Ray, M. K., Lokhande, A., Salunke, D. (2016). A PAPR Reduction of OFDM Signal by using Nonlinear Companding Transform, International Journal of Engineering Science, 2893.

[24] AL-Hashmi, Z. S. H. (2015). An Overview : Peak to Average Power Ratio (PAPR) in OFDM system using some new PAPR techniques (with matlab code), Engineering, (October 3).

[25] Bindu, B. A. H., Chandrika, O. M. (2016). Combined DCT and Companding for PAPR Reduction in OFDM Signals, International Journal for Innovative Research in Science and Technology, 2, 730-735.

[26] Rao, M. N., Naveen, V. J., Kishore, K. K. (2016). Performance analysis of Piecewise linear Companding with various precoders for PAPR Reduction of OFDM Signals.

[27] Ajay, T., Krishna, K. M. (2016). A Hybrid technique for PAPR Reduction of OFDM using DHT Precoding with Piecewise Linear Companding, 1 (8).

[28] Ghahremani, R., Shayesteh, M. G. (2014). BER performance improvement and PAPR reduction in OFDM systems based on combined DHT and $\mu$ - Law companding, In: Electrical Engineering (ICEE), 2014 22nd Iranian Conference on, 2014, 1483-1487.

[29] Virdi, J., Kumar, S. (2013). PAPR Reduction Based on Precoding Techniques with Companding in OFDM Systems, International Journal of Scientific \& Engineering Research, 4, 1064-1070.

[30] Elmaroud, B., Faqihi, A., Abbad, M., Aboutajdine, D. (2014). PAPR reduction of FBMC signals by combining exponential companding and hadamard transforms, In: Networks, Computers and Communications, the 2014 International Symposium on, 2014, 1-4. 